\title{
Role of immune checkpoint blockers in patients with EGFR mutation
}

\author{
Jeffrey Ward, Daniel Morgensztern \\ Division of Oncology, Washington University School of Medicine, St. Louis, MO 63110, USA \\ Correspondence to: Daniel Morgensztern, MD. Associate Professor of Medicine, Division of Oncology, Washington University School of Medicine, \\ 660 South Euclid Avenue, Campus Box 8056, St. Louis, MO 63110, USA. Email: danielmorgensztern@wustl.edu. \\ Comment on: Lisberg A, Cummings A, Goldman JW, et al. A Phase II Study of Pembrolizumab in EGFR-Mutant, PD-L1+, Tyrosine Kinase Inhibitor \\ Naïve Patients With Advanced NSCLC. J Thorac Oncol 2018;13:1138-45.
}

Submitted Aug 18, 2018. Accepted for publication Sep 19, 2018.

doi: $10.21037 /$ tlcr.2018.09.13

View this article at: http://dx.doi.org/10.21037/tlcr.2018.09.13

Single agent epidermal growth factor receptor (EGFR) tyrosine kinase inhibitors (TKIs) are the current standard of care for patients with advanced stage non-small cell lung cancer (NSCLC) harboring sensitizing mutations in the EGFR gene, with response rates and progression-free survival (PFS) consistently better than chemotherapy $(1,2)$. Nevertheless, despite the high initial responses, virtually all patients develop tumor progression with a median PFS of less than 12 months with first or second generation EGFR TKIs and 18.9 months with the third generation osimertinib (3). Therefore, there is a need for new therapies with more durable responses in this population of predominantly younger patients with less exposure to tobacco smoking.

Blockade of programmed death 1 (PD-1) or its ligand (PD-L1) has been associated with prolonged responses in a small percentage of patients with NSCLC. In the phase I CA209-003, the study with the longest followup available, the estimated 5 -year overall survival (OS) for patients treated with nivolumab was $16 \%$, with 12 patients not requiring additional therapy for 3 or more years after completing up to 96 weeks of treatment (4).

Immune checkpoint blockade (ICB) antibodies in patients with $E G F R$ mutation has been associated with decreased efficacy compared to patients with wild type tumors. In a meta-analysis of three randomized studies comparing nivolumab, pembrolizumab or atezolizumab to docetaxel, there was no improvement in OS for ICB, unlike the EGFR wild type counterpart (5). Furthermore, in the Italian nivolumab expanded access study, the response rates were lower in EGFR mutant patients than in wild type (8.8\% vs. 19.0\%) (6). Among never-smokers with EGFR mutation, only one out of 51 patients (2\%) achieved tumor response.

In the phase 1 Keynote-001 trial evaluating the role of pembrolizumab in advanced stage NSCLC, 74 out of 478 patients (15.5\%) had EGFR mutation and 94 (19.7\%) were treatment-naive (7). Lisberg and colleagues (8) evaluated the role of pembrolizumab in patients with EGFR mutation, PD-L1 positive defined as $\geq 1 \%$ tumor membranous staining using the $22 \mathrm{C} 3$ antibody, TKI naïve, advanced stage NSCLC. The rationale for the study was the University of California, Los Angeles (UCLA) experience on the Keynote-001 where, among the four previously untreated patients with sensitizing EGFR mutation, the objective response rate (ORR) and median PFS were 50\% and 5.8 months respectively. In contrast, among the 26 $E G F R$-mutant patients previously treated with TKI, the ORR and median PFS were $4 \%$ and 1.9 months. The study further selected patients by including only those with PD-L1 positive, which is a known predictive factor for benefit from pembrolizumab (9). The primary objective of the study was ORR, whereas secondary endpoints included safety, PFS and OS. The study was designed to show an ORR $\geq 26 \%$. Eligible patients received $200 \mathrm{mg}$ of pembrolizumab every three weeks for up to 35 cycles. After completion of pembrolizumab, patients were followed for evaluation of subsequent EGFR TKI safety and efficacy as additional secondary endpoints. Study enrollment was discontinued after 11 of the planned 25 patients due to futility. Of the 11 patients, 7 (64\%) had either an EGFR exon 19 deletion or an L858R mutation, and 8 (73\%) had a PD-L1 proportion 
score of $\geq 50 \%$. The only patient that achieved objective response, had PD-L1 score of $80 \%$ but was later found not to have EGFR mutation, leading to an ORR of $0 \%$ among the 10 patients with confirmed eligibility criteria. Among these patients, the median PFS was 4 months and the median OS was not reached after a follow-up of 7.5 months. More concerning than the lack of responses was the safety profile. Although there were no grade 4 or 5 toxicities during treatment with pembrolizumab, 6 out of 7 patients (86\%) that received EGFR TKI as the next line of therapy experienced adverse events presumed to be associated with the TKI, including grade 3 elevation of liver enzymes and a grade 5 pneumonitis, with the latter occurring 89 days after starting erlotinib.

Despite the small number of patients enrolled, this study adds to the growing evidence that combinations with ICBs and TKIs, administered either concurrently or sequentially in the first-line setting, may lead to severe and unexpected toxicities. In the phase $1 \mathrm{~b}$ TATOON trial, were patients with EGFR mutation received first-line concurrent osimertinib and durvalumab, the incidence of interstitial lung disease was $38 \%$ leading to discontinuation of the study (10). The CheckMate 370 study, where patients with anaplastic lymphoma kinase (ALK) translocation positive patients were treated with first-line nivolumab plus crizotinib, 5 out of the initial 13 patients (38\%) developed severe hepatic toxicities, leading to the early discontinuation of the study (11). In contrast, the combination of nivolumab with erlotinib in patients with EGFR mutations was well tolerated in a phase 1 study involving 21 patients (12). In this study, however, 20 patients were previously treated with TKIs. The results in this population were promising with ORR of $15 \%$, disease control rate of $65 \%$, median PFS of 5.1 months and median OS of 18.7 months.

There are several explanations for the decreased efficacy of pembrolizumab in patients with EGFR mutant tumors. Perhaps the most compelling explanation is the fewer number of mutations and neoantigens in patients with EGFR-driven tumors, which are commonly never-smokers or light-smokers as shown in Lisberg's study (8), where 6 out of the 10 eligible patients were never-smokers and 4 were prior smokers. Neoantigens, altered peptides that arise due to mutations accumulated during carcinogenesis, can serve as the targets for the adaptive immune response occurring with the use of checkpoint blockade therapies (13). Adenocarcinomas in never smokers express fewer mutations compared to smokers (14) and mutation load has been associated with increased efficacy from ICBs (15). It is unclear whether the lower mutation load observed in patients that are never smokers or EGFR-mutant is still sufficient to elicit enough neoantigen-specific CD8+ T-cells to derive a prolonged clinical benefit. There may also be additional tumor intrinsic mechanisms, occurring in the presence of EGFR mutations, which prevent an adequate immune response. In a retrospective analysis, patients with EGFR mutations or ALK translocations had low rates of concurrent PD-L1 expression and CD8+ tumor-infiltrating lymphocytes (TILs) in the tumor microenvironment (16). Since both POD-L1 and CD8+ TILs are associated with responses to ICB, this finding may at least in part explain the lack of benefit from ICBs in this patient population (17).

In summary, the standard of care initial therapy for patients with sensitizing EGFR mutations remains with single agent EGFR TKI. Although the use of ICBs in patients previously treated with EGFR TKIs may be safer, and the addition of nivolumab to erlotinib at the time of tumor progression during treatment with the latter was well tolerated, initial treatment with ICBs either concurrently or followed by TKIs is not indicated at this time due to the increased toxicity. Novel combinations are needed to provide durable responses, improve survival and possibly lead to cure in patients with EGFR mutation.

\section{Acknowledgements}

None.

\section{Footnote}

Conflicts of Interest: J Ward-Consulting or Advisory Role: Boehringer Ingelheim; D Morgensztern-Consulting or Advisory Role: Celgene, Bristol-Myers Squibb, Abbvie.

\section{References}

1. Masters GA, Temin S, Azzoli CG, et al. Systemic Therapy for Stage IV Non-Small-Cell Lung Cancer: American Society of Clinical Oncology Clinical Practice Guideline Update. J Clin Oncol 2015;33:3488-515.

2. Ettinger DS, Wood DE, Aisner DL, et al. Non-Small Cell Lung Cancer, Version 5.2017, NCCN Clinical Practice Guidelines in Oncology. J Natl Compr Canc Netw 2017;15:504-35.

3. Soria JC, Ohe Y, Vansteenkiste J, et al. Osimertinib in Untreated EGFR-Mutated Advanced Non-Small-Cell Lung Cancer. N Engl J Med 2018;378:113-25. 
4. Gettinger S, Horn L, Jackman D, et al. Five-Year FollowUp of Nivolumab in Previously Treated Advanced NonSmall-Cell Lung Cancer: Results From the CA209-003 Study. J Clin Oncol 2018;36:1675-84.

5. Lee CK, Man J, Lord S, et al. Checkpoint Inhibitors in Metastatic EGFR-Mutated Non-Small Cell Lung Cancer-A Meta-Analysis. J Thorac Oncol 2017;12:403-7.

6. Garassino MC, Gelibter AJ, Grossi F, et al. Italian Nivolumab Expanded Access Program in Nonsquamous Non-Small Cell Lung Cancer Patients: Results in NeverSmokers and EGFR-Mutant Patients. J Thorac Oncol 2018;13:1146-55.

7. Garon EB, Rizvi NA, Hui R, et al. Pembrolizumab for the treatment of non-small-cell lung cancer. N Engl J Med 2015;372:2018-28.

8. Lisberg A, Cummings A, Goldman JW, et al. A Phase II Study of Pembrolizumab in EGFR-Mutant, PD-L1+, Tyrosine Kinase Inhibitor Naive Patients With Advanced NSCLC. J Thorac Oncol 2018;13:1138-45.

9. Herbst RS, Baas P, Kim DW, et al. Pembrolizumab versus docetaxel for previously treated, PD-L1-positive, advanced non-small-cell lung cancer (KEYNOTE-010): a randomised controlled trial. Lancet 2016;387:1540-50.

10. Ahn MJ, Yang J, Yu H, et al. 136O: Osimertinib combined with durvalumab in EGFR-mutant non-small cell lung cancer: Results from the TATTON phase Ib trial. J Thorac Oncol 2016;11:S115.

Cite this article as: Ward J, Morgensztern D. Role of immune checkpoint blockers in patients with EGFR mutation. Transl Lung Cancer Res 2018;7(Suppl 4):S385-S387. doi: 10.21037/ tlcr.2018.09.13
11. Spigel DR, Reynolds C, Waterhouse D, et al. Phase $1 / 2$ Study of the Safety and Tolerability of Nivolumab Plus Crizotinib for the First-Line Treatment of Anaplastic Lymphoma Kinase Translocation - Positive Advanced Non-Small Cell Lung Cancer (CheckMate 370). J Thorac Oncol 2018;13:682-8.

12. Gettinger S, Hellmann MD, Chow LQM, et al. Nivolumab Plus Erlotinib in Patients With EGFR-Mutant Advanced NSCLC. J Thorac Oncol 2018;13:1363-72.

13. Gubin MM, Zhang X, Schuster H, et al. Checkpoint blockade cancer immunotherapy targets tumour-specific mutant antigens. Nature 2014;515:577-81.

14. Govindan R, Ding L, Griffith M, et al. Genomic landscape of non-small cell lung cancer in smokers and neversmokers. Cell 2012;150:1121-34.

15. Rizvi NA, Hellmann MD, Snyder A, et al. Cancer immunology. Mutational landscape determines sensitivity to PD-1 blockade in non-small cell lung cancer. Science 2015;348:124-8.

16. Gainor JF, Shaw AT, Sequist LV, et al. EGFR Mutations and ALK Rearrangements Are Associated with Low Response Rates to PD-1 Pathway Blockade in Non-Small Cell Lung Cancer: A Retrospective Analysis. Clin Cancer Res 2016;22:4585-93.

17. Teng $M W$, Ngiow SF, Ribas A, et al. Classifying Cancers Based on T-cell Infiltration and PD-L1. Cancer Res 2015;75:2139-45. 